\title{
Novel Methods of Lymph Node Evaluation for Predicting the Prognosis of Colorectal Cancer Patients with Inadequate Lymph Node Harvest
}

\section{Taek Soo Kwon, MD \\ Sung Bong Choi, MD \\ Yoon Suk Lee, MD, PhD \\ Jun-Gi Kim, MD, PhD \\ Seong Taek Oh, MD, PhD \\ In Kyu Lee, MD, PhD}

Department of Surgery, College of Medicine,

The Catholic University of Korea, Seoul, Korea

Correspondence: In Kyu Lee, MD, PhD

Department of Surgery,

Seoul St. Mary's Hospital, College of Medicine,

The Catholic University of Korea,

222 Banpo-daero, Seocho-gu, Seoul 06591, Korea

Tel: $82-2-2258-6104$

Fax: 82-2-595-2822

E-mail: cmcgslee@catholic.ac.kr

Received October 31, 2014

Accepted March 7, 2015

Published Online April 15, 2015

${ }^{*}$ Taek Soo Kwon and Sung Bong Choi contributed equally to this work.

\begin{abstract}
Purpose
Lymph node metastasis is an important factor for predicting the prognosis of colorectal cancer patients. However, approximately $60 \%$ of patients do not receive adequate lymph node evaluation (less than 12 lymph nodes). In this study, we identified a more effective tool for predicting the prognosis of patients who received inadequate lymph node evaluation.
\end{abstract}

\section{Materials and Methods}

The number of metastatic lymph nodes, total number of lymph nodes examined, number of negative metastatic lymph nodes (NL), lymph node ratio (LR), and the number of apical lymph nodes (APL) were examined, and the prognostic impact of these parameters was examined in patients with colorectal cancer who underwent surgery from January 2004 to December 2011. In total, 806 people were analyzed retrospectively.

\section{Results}

In comparison of different lymph node analysis methods for rectal cancer patients who did not receive adequate lymph node dissection, the LR showed a significant difference in overall survival (OS) and the APL predicted a significant difference in disease-free survival (DFS). In the case of colon cancer patients who did not receive adequate lymph node dissection, LR predicted a significant difference in DFS and OS, and the APL predicted a significant difference in DFS.

\section{Conclusion}

If patients did not receive adequate lymph node evaluation, the LR and NL were useful parameters to complement $N$ stage for predicting $O S$ in colon cancer, whereas $L R$ was complementary for rectal cancer. The APL could be used for prediction of DFS in all patients.

\section{Introduction}

Colorectal cancer (CRC) prognosis is predicted by TNM staging. The recent American Joint Committee on Cancer (AJCC) manual also recognizes the following prognostic factors: preoperatively elevated serum carcinoembryonic antigen, satellite tumor deposit, tumor regression grade, circumferential resection margin, microsatellite instability, perineural invasion and KRAS mutational status [1].

\section{Key words}

Colorectal neoplasms, Prognosis, Lymph nodes,

Negative lymph nodes, Lymph node ratio
Lymph node metastasis ( $\mathrm{N}$ stage) is the single most important prognostic factor in CRC [2]. Baxter et al. [3] used data from the National Cancer Institute's Surveillance, Epidemiology, and End Results (SEER) program to determine the proportion of CRC patients in the United States who received adequate lymph node evaluation. Of 116,695 adults with CRC diagnosed from 1988 through 2001 who underwent radical surgery and did not receive neoadjuvant radiation (i.e., at least 12 lymph nodes were examined), only $37 \%$ of the patients received adequate lymph node evaluation. For 
example, the 5-year survival rate for patients classified with stage II disease was $64 \%$ when one or two lymph nodes were sampled, it increased to $86 \%$ when more than 25 nodes were sampled. In conclusion, $63 \%$ of patients with CRC did not receive an accurate staging evaluation because of inadequate lymph node dissection, and these patients likely did not receive the appropriate treatment.

Therefore, the purpose of this paper was to determine whether lymph node metastasis represents a complementary method to the TNM stage method for accurate prediction of prognosis (the number of lymph node metastases) in patients who did not receive adequate lymph node evaluation. nation were placed in the inadequate lymph node evaluation group (IAL group). We analyzed the statistical significance between these three groups.

\section{Statistical analysis}

Five-year disease-free survival (DFS) and 5-year overall survival (OS) were calculated by the Kaplan-Meier method using SPSS ver. 18.0 (SPSS Inc., Chicago, IL). A p-value $<0.05$ was considered statistically significant.

\section{Results}

\section{Materials and Methods}

\section{Study population}

A total of 806 patients who underwent surgery for CRC in Yeouido St. Mary's Hospital from January 2004 to December 2011 were analyzed retrospectively, and the data were collected prospectively.

In total, 692 patients were analyzed, including those with adenocarcinoma. Patients who did not undergo colorectal resection, who underwent transanal excision and those whose tumor location was not labeled correctly in the documentation were excluded.

The Yeouido St. Mary's Hospital Institutional Review Board approved this analysis (SC13OISI0180).

\section{Lymph node analysis}

To date, there are five types of lymph nodes that could be used for analysis: metastatic lymph nodes (ML), total lymph nodes examined (TL) [4], negative metastatic lymph nodes (NL) [5], lymph node ratio (LR=ML/TL) [6], and apical lymph nodes (APL) [7].

ML were analyzed according to the $\mathrm{N}$ stage, and TL were classified as less than 10,11-20, 21-40, and $\geq 41$. NL was scored as $\leq 3,4-6,7-12$, or $\geq 13$. The LR was classified as $<0.2$, $0.2-0.5$, or $\geq 0.5$. APL were measured in approximately 359 patients from whom $2 \mathrm{~cm}$ of soft tissue was collected from the origin of the primary feeding arterial vessel (e.g., inferior mesenteric artery, right colic artery, or ileocolic artery), and the presence or absence of ML was noted.

All patients, regardless of the examined lymph node count, were included in the all group (A group). Patients in whom at least 12 lymph nodes were examined were included in the adequate lymph nodes evaluation group (AL group). Patients who did not receive adequate lymph nodes exami-

\section{Patient characteristics}

A total of 692 patients with CRC were included in this study; 489 had rectal cancer and 203 were colon cancer patients. The mean ages of the colon cancer and rectal cancer patients were 63.2 and 61.1 years old, respectively.

The male to female ratio of the colon cancer population was $284(58.1 \%)$ to $205(41.9 \%)$, and that for rectal cancer patients was $134(66 \%)$ to $69(34 \%)$. APL were harvested from 252 patients $(51.5 \%)$ with colon cancer and 107 patients $(52.7 \%)$ with rectal cancer. Inadequate lymph node evaluation occurred in $138(28.2 \%)$ colon cancer and $104(51.2 \%)$ rectal cancer patients (Table 1).

The mean follow-up period was 83.5 months. In total, the 5 -year DFS was $67.5 \%$ and the 5 -year OS was $85.2 \%$. The 5-year DFS for each stage was as follows: stage I, 92.9\%; stage II, 73.7\%; stage III, 56.2\%; and stage IV, $24.9 \%$. The 5-year OS for each stage was as follows: stage I, $94.2 \%$; stage II, $93.6 \%$; stage III, $83.6 \%$; and stage IV, $30.0 \%$.

\section{Various lymph node analysis methods}

\section{1) Metastatic lymph node (existing $\mathrm{N}$ stage)}

Univariate analysis was performed for analysis of the impact of ML on patient outcome. In the rectum, OS did not differ statistically (A group, $\mathrm{p}=0.062$; AL group $\mathrm{p}=0.241$ ). The number of $\mathrm{ML}$, especially for patients who received inappropriate lymph node dissection (IAL group), did not represent a significant difference in DFS $(\mathrm{p}=0.339)$ and $\mathrm{OS}(\mathrm{p}=0.146)$ for rectal cancer patients and OS $(\mathrm{p}=0.129)$ for colon cancer patients (Table 2).

\section{2) Total lymph node}

The number of TL was not statistically significant for any 
parameter, except OS ( $\mathrm{p}=0.042$ ) in the AL group of colon cancer patients. There was no greater efficacy than the existing $\mathrm{N}$ stage system (Table 2). In the subgroup analysis for stage I, OS ( $p=0.049)$ was significantly different in the AL group of CRC (Table 3). For stage II and III, TL was not statistically significant. For stage IV, the OS of the A group ( $p=0.02)$ and $\operatorname{AL}$ group $(\mathrm{p}=0.041)$ of $C R C$, and the A group $(\mathrm{p}=0.0028)$ and AL group of colon cancer patients showed statistical significance (Table 3). Lymph node harvest (LNH) count $(\leq 10$, 11$20,21-40$, or $\geq 41$ ) affected the OS in the stage IV CRC and colon groups. Thus, lymph node dissection improved OS in stage IV colon cancer.

\section{3) Negative metastatic lymph node}

NL was as statistically significant as the conventional $\mathrm{N}$ stage system, and it showed a statistically significant result for the OS $(p=0.016)$ of patients with colon cancer who received improper node dissection (IAL group) (Table 2, Fig. 1).

\section{4) Metastatic lymph node ratio}

LR was more useful than the conventional N stage system, as it showed statistically significant efficacy for OS ( $p<0.001)$ in the IAL group of colon cancer and for OS in rectal cancer (A group, $\mathrm{p}=0.026$; AL group, $\mathrm{p}=0.031$; and IAL group, $\mathrm{p}=0.003)$ (Table 2, Fig. 1$)$.

In the subgroup analysis, stage I, II, and early cancer (node negative cancer) were meaningless for LR because no ML were present. In stage III, IV, and advanced cancer (node positive cancer), the DFS for colon cancer patients who received inappropriate lymph node dissection (IAL group) was statistically significant for LR but not for $\mathrm{N}$ stage (Table 3 ).

\section{5) Apical lymph node}

APL showed significance for DFS ( $\mathrm{p}=0.036)$ of the IAL group of rectal cancer, which was not predicted by the conventional $\mathrm{N}$ stage system. APL showed efficacy in predicting DFS in all patients, except the AL group of colon cancer (Table 2).

\section{6) Comparison of the different lymph node analysis} methods

ML (conventional N stage) did not show significant differences in the OS of rectal cancer patients. In particular, if patients received inadequate lymph node dissection (IAL group), OS in colon cancer and DFS and OS in rectal cancer were not statistically different.

These limitations were overcome using NL and LR to predict the OS of colon cancer patients and the LR for the OS of
Table 1. Patient characteristics

\begin{tabular}{|c|c|c|}
\hline Characteristic & Colon $(n=489)$ & Rectum (n=203) \\
\hline Mean age (yr) & 63.21 & 61.1 \\
\hline \multicolumn{3}{|l|}{ Sex } \\
\hline Male & $284(58.1)$ & $134(66)$ \\
\hline Female & 205 (41.9) & $69(34)$ \\
\hline \multicolumn{3}{|l|}{ TNM stage } \\
\hline I & $99(20.2)$ & $48(23.6)$ \\
\hline II & $158(32.3)$ & $63(31)$ \\
\hline III & $167(34.2)$ & $73(36)$ \\
\hline IV & 65 (13.3) & $19(9.40)$ \\
\hline Early (stage I, II) & $257(52.6)$ & $111(54.7)$ \\
\hline Advance (stage III, IV) & $232(47.4)$ & $92(45.3)$ \\
\hline \multicolumn{3}{|l|}{ Metastatic LN } \\
\hline N0 & $268(54.8)$ & $117(57.6)$ \\
\hline N1 & $125(25.6)$ & $51(25.1)$ \\
\hline N2 & $96(19.6)$ & $35(17.2)$ \\
\hline \multicolumn{3}{|l|}{ Total LN } \\
\hline $0-10$ & $114(23.3)$ & $95(46.8)$ \\
\hline $11-20$ & $180(36.8)$ & $74(36.5)$ \\
\hline $21-40$ & $165(33.7)$ & $30(14.8)$ \\
\hline$\geq 41$ & $30(6.1)$ & $4(2.0)$ \\
\hline \multicolumn{3}{|l|}{ Negative LN } \\
\hline$\leq 3$ & $31(6.3)$ & $31(15.3)$ \\
\hline $4-6$ & $56(11.5)$ & $31(15.3)$ \\
\hline $7-12$ & $120(24.5)$ & $71(35.0)$ \\
\hline$\geq 13$ & $282(57.7)$ & $70(34.5)$ \\
\hline \multicolumn{3}{|l|}{$\begin{array}{l}\mathrm{LN} \text { ratio } \\
\qquad(\mathrm{n}=682 \text {, except } \mathrm{TL}=0)\end{array}$} \\
\hline$<0.2$ & $388(80.3)$ & $146(73.4)$ \\
\hline 0.2 to $<0.5$ & $62(12.8)$ & $32(16.1)$ \\
\hline$\geq 0.5$ & $33(6.8)$ & $21(10.5)$ \\
\hline \multicolumn{3}{|l|}{ Apical lymph node } \\
\hline Negative & $239(94.8)$ & $102(95.3)$ \\
\hline Positive & $13(5.2)$ & $5(4.7)$ \\
\hline Apical LN harvest & $252(51.5)$ & $107(52.7)$ \\
\hline \multicolumn{3}{|l|}{ LN dissection } \\
\hline Inadequate $(\mathrm{LN}<12)$ & $138(28.2)$ & $104(51.2)$ \\
\hline Adequate $(\mathrm{LN} \geq 12)$ & $351(71.8)$ & $99(48.8)$ \\
\hline
\end{tabular}

Values are presented as number (\%). Colon, colon cancer; rectum, rectal cancer; LN, lymph node.

rectal cancer patients. APL accurately predicted DFS in the IAL group and in the OS of all patients. 
Table 2. Comparison of lymph node analysis

\begin{tabular}{|c|c|c|c|c|c|c|}
\hline \multirow{3}{*}{ All stage } & \multicolumn{6}{|c|}{ p-value } \\
\hline & \multicolumn{2}{|c|}{ CRC } & \multicolumn{2}{|c|}{ Colon } & \multicolumn{2}{|c|}{ Rectum } \\
\hline & DFS & OS & DFS & OS & DFS & OS \\
\hline \multicolumn{7}{|l|}{ N stage } \\
\hline A group & $<0.001$ & $<0.001$ & $<0.001$ & $<0.001$ & 0.002 & 0.062 \\
\hline AL group & $<0.001$ & $<0.001$ & $<0.001$ & $<0.001$ & 0.001 & 0.241 \\
\hline IAL group & $<0.001$ & 0.03 & $<0.001$ & 0.129 & 0.339 & 0.146 \\
\hline \multicolumn{7}{|l|}{ Total LN } \\
\hline A group & 0.818 & 0.082 & 0.872 & 0.056 & 0.524 & 0.678 \\
\hline AL group & 0.857 & 0.062 & 0.781 & 0.042 & 0.807 & 0.467 \\
\hline IAL group & 0.32 & 0.345 & 0.905 & 0.569 & 0.27 & 0.335 \\
\hline \multicolumn{7}{|l|}{ Negative LN } \\
\hline A group & 0.002 & 0.001 & 0.003 & 0.001 & 0.053 & 0.418 \\
\hline AL group & $<0.001$ & $<0.001$ & $<0.001$ & $<0.001$ & 0.002 & 0.696 \\
\hline IAL group & 0.048 & 0.002 & 0.008 & 0.016 & 0.539 & 0.153 \\
\hline \multicolumn{7}{|l|}{$\mathrm{LN}$ ratio } \\
\hline A group & $<0.001$ & $<0.001$ & $<0.001$ & $<0.001$ & 0.027 & 0.026 \\
\hline AL group & $<0.001$ & $<0.001$ & $<0.001$ & $<0.001$ & 0.008 & 0.031 \\
\hline IAL group & $<0.001$ & $<0.001$ & $<0.001$ & $<0.001$ & 0.686 & 0.003 \\
\hline \multicolumn{7}{|l|}{ Apical LN } \\
\hline A group & $<0.001$ & 0.026 & 0.026 & 0.005 & $<0.001$ & 0.68 \\
\hline AL group & 0.001 & 0.013 & 0.095 & 0.004 & $<0.001$ & 0.81 \\
\hline IAL group & 0.002 & 0.693 & 0.044 & 0.88 & 0.036 & 0.747 \\
\hline
\end{tabular}

Bold data indicate a comparison with other methods of lymph node analysis that does not represent a significant difference compared to the N stage. CRC, colorectal cancer; colon, colon cancer; rectum, rectal cancer; DFS, disease-free survival; OS, overall survival; A group, all patients; AL group, adequate lymph node evaluation group; IAL group, inadequate lymph node evaluation group; LN, lymph nodes. Metastatic LN, N stage: 0, 1 (1-3), 2 ( $\geq 4)$; total LN: the number of lymph nodes examined $(\leq 10,11-20,21-40, \geq 41)$; negative LN: the number of negative lymph nodes $(\leq 3,4-6,7-12, \geq 13)$; LN ratio: $<0.2,0.2-0.5, \geq 0.5$; apical LN: positive vs. negative.

\section{Discussion}

Regarding prognostic factors in CRC, the College of American Pathologists consensus statement in 1999 by Compton et al. [8] stated that lymph node metastasis of CRC was definitely proven to be prognostically important (category I). However, due to insufficient data, the use of various analysis methods is still recommended. One limitation of the current $\mathrm{N}$ stage system is that it cannot provide a precise prognosis for patients who did not have over 12 lymph nodes dissected. Thus, we sought to identify another lymph node analysis method to complement the $\mathrm{N}$ stage system.

In our study, inadequate lymph node evaluation occurred in 138 colon cancer $(28.2 \%)$ and 104 rectal cancer patients (51.2\%). According to a Norwegian national cohort study of 2011, adequate LNH rate from 2007 to 2008 was $69.6 \%$ for colon cancers more than $15 \mathrm{~cm}$ above the anal verge. Adequate LNH rate was $84.9 \%$ in stage II colon cancer of Cleveland Clinic (Cleveland, $\mathrm{OH}$ ). Adequate $\mathrm{LNH}$ rate ranges from $31 \%$ to $84.9 \%$ in CRCs, and risk factors for poor $\mathrm{LNH}$ include early stage CRCs and neoadjuvant concurrent chemoradiotherapy [9-11]. In addition, the rate of adequate LNHs has shown a drastic increase since the National Comprehensive Cancer Network guideline referred to $\mathrm{LNH}$ below 12 nodes as a high risk group [12]. In accordance, our study showed $43.8 \%$ inadequate LNHs until 2008, while it decreased to $18.4 \%$ from 2009 to 2011 . Conventional surgery and low ligation was the primary surgical method of our institution until 2008, but conversion to laparoscopic surgery and high ligation as the primary method thereafter has increased the number of adequate LNHs.

ML was used as the reference, and the patients were subdivided further and analyzed statistically. In the case of stage 


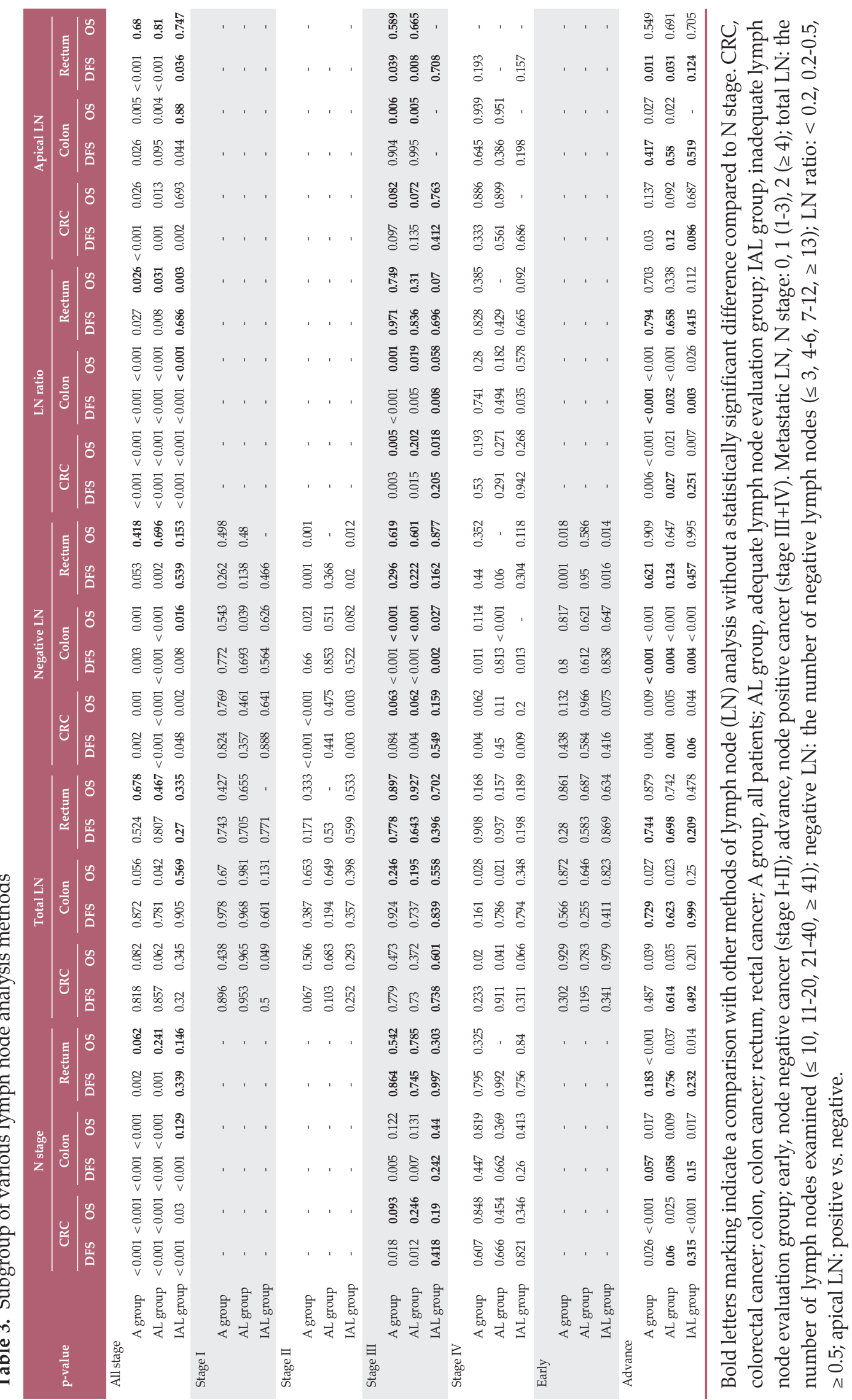



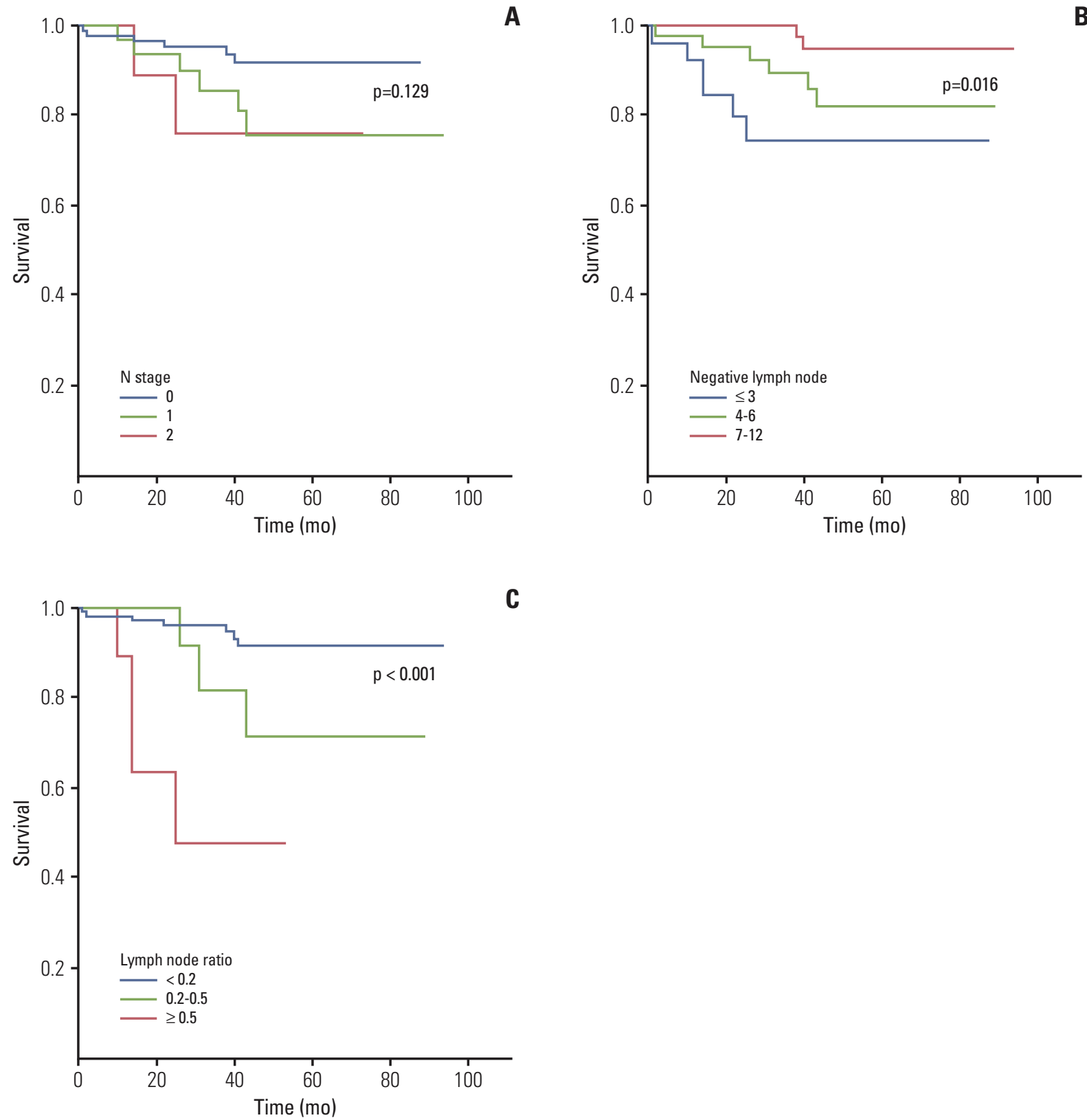

C

Fig. 1. Comparison of $\mathrm{N}$ stage (A), negative lymph node (B), and lymph node ratio (C) with overall survival of colon cancer patients who received inadequate lymph node evaluation.

I, II and early cancer, there was no value because the patients were node negative. For stage III of the IAL group of ML, no statistically significant differences were observed in the following groups: DFS $(\mathrm{p}=0.418)$ and $\mathrm{OS}(\mathrm{p}=0.19)$ of CRC patients, DFS $(\mathrm{p}=0.242)$ and OS $(\mathrm{p}=0.44)$ of colon cancer patients, and DFS $(\mathrm{p}=0.992)$ and OS $(\mathrm{p}=0.303)$ of patients with rectal cancer. No statistically significant differences were observed for stage IV of ML. In advanced cancer (node positive), the ML was statistically significant for OS but not DFS (Table 3).

Chang et al. [4], who systematically reviewed 17 studies (including two nested cohort studies) regarding lymph node evaluation after the curative resection of colon cancer, concluded that the number of lymph nodes evaluated after surgical resection was positively associated with the survival of patients with stage II and stage III colon cancer [4]. The effect 

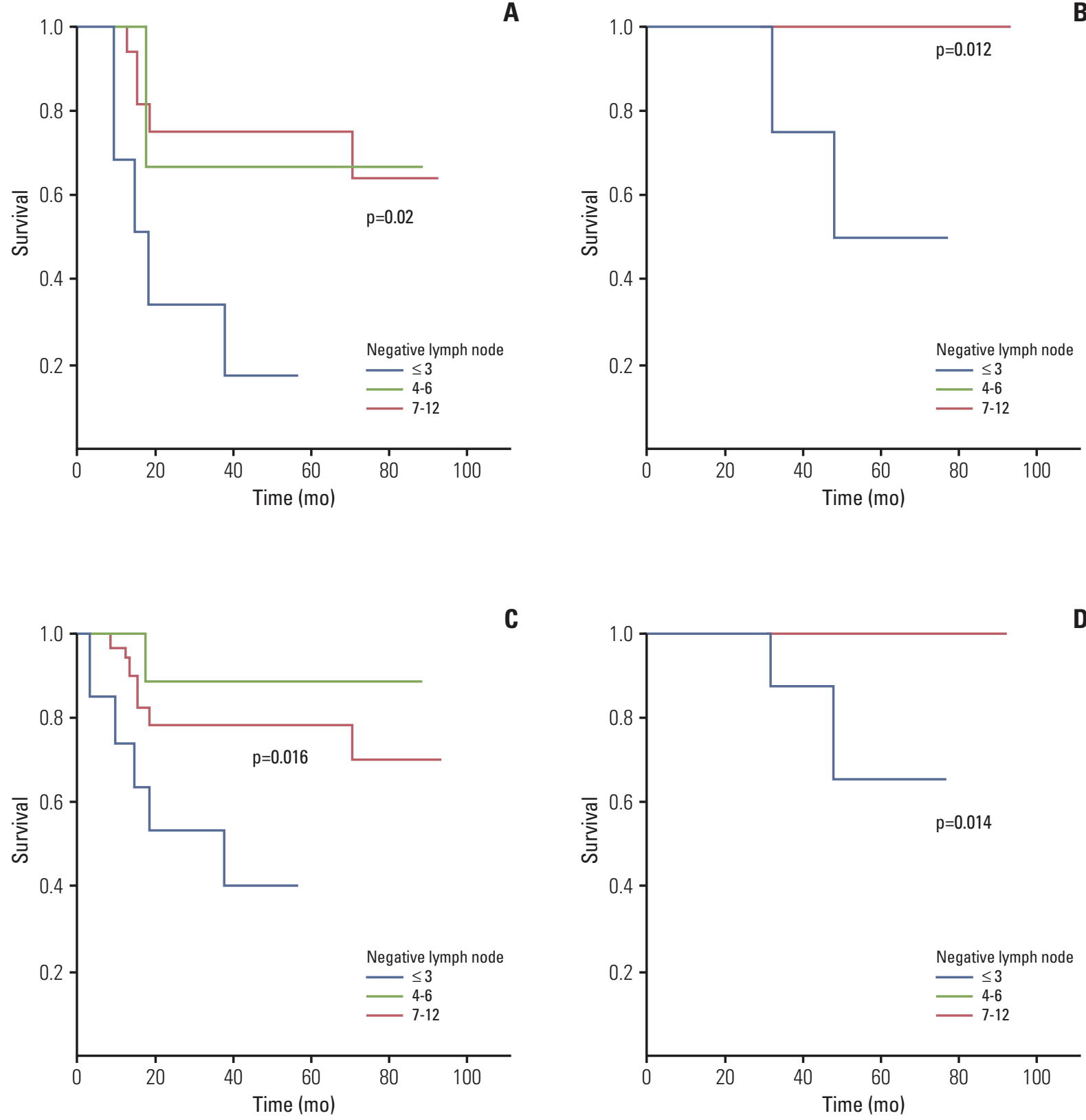

Fig. 2. Efficacy of negative lymph node in predicting disease-free survival (DFS) (A) and overall survival (OS) (B) of rectal cancer patients in stage II, DFS (C) and OS (D) in early rectal cancer, who received inadequate lymph node evaluation.

of increased lymph node recovery on improving survival may not be fully explained by improved staging accuracy alone. Chang et al. [4] also described various TL classification criteria; however, we used two criteria in our data analysis: $(1) \leq 10,11-20,21-40$, and $\geq 41$ and $(2) \leq 11$ and $\geq 12$. The former criteria showed statistical significance, therefore we used those criteria, but they showed no additional utility compared to ML.
Ogino et al. [5] reported that patients with 0-3, 7-12, and $\geq 13$ negative lymph nodes showed a significant reduction in cancer-specific and overall mortality. Using a similar system, we performed statistical analysis of patient parameters based on different subdivisions. In stage I, only the OS $(p=0.039)$ of the AL group of colon cancer was significant. For stage II in the IAL group, NL showed a statistically significant difference in the DFS $(p=0.003)$ and OS $(p=0.003)$ 
of CRC patients and DFS ( $\mathrm{p}=0.02)$ and OS $(\mathrm{p}=0.012)$ of rectal cancer patients. Importantly, it predicted a significant decrease in the survival of patients with three or less lymph node compared the four to six and 7 to 11 lymph node count groups (Table 3, Fig. 2). Therefore, for stage II rectal cancer patients, NL count (i.e., the LNH number was three or less) indicated that short-term follow-up and additional treatment were necessary.

For the early IAL rectal cancer group (i.e., node negative patients who underwent polypectomy or endoscopic submucosal dissection before surgery due to incorrect $\mathrm{T}$ staging of the primary tumor), NL showed a statistical value in predicting DFS $(\mathrm{p}=0.016)$ and OS $(\mathrm{p}=0.014)$ (Table 3, Fig. 2). Therefore, in patients with three or less total lymph node, there was a difference in the DFS and OS, and close observation is warranted.

Johnson et al. [13] reported that increasing the negative lymph node count is independently associated with improved long-term survival in stage IIIB and IIIC colon cancer but not stage IIIA. They also divided patients into subgroups of those with $\leq 3,4-7,8-12$, or $\geq 13$ negative lymph nodes. Cumulative 5 -year cancer mortality was $27 \%$ in stage IIIB patients with $\geq 13$ negative nodes identified versus $45 \%$ in those with three or fewer negative lymph nodes evaluated $(p<0.001)$. In patients with stage IIIC cancer, those with $\geq 13$ negative nodes had a 5 -year mortality of $42 \%$ versus $65 \%$ in those with $\leq 3$ negative lymph nodes evaluated $(\mathrm{p}<0.001)$ [13].

For stage III in the IAL group, ML was not statistically meaningful. However, NL was statistically significant in predicting DFS ( $\mathrm{p}=0.002)$ and OS $(\mathrm{p}=0.0027)$ for colon cancer patients (Table 3 ). Therefore, for patients in the IAL group of colon cancer, NL (with 3 or less, 4-6, and 7-11) complemented the existing $\mathrm{N}$ stage.

For stage IV cancer, the DFS of the A group $(\mathrm{p}=0.004)$ and IAL group ( $\mathrm{p}=0.009$ ) of $\mathrm{CRC}$, and the DFS of the A group $(\mathrm{p}=0.011)$ and IAL group $(\mathrm{p}=0.013)$ and the OS of the AL group ( $\mathrm{p}<0.001$ ) of colon cancer patients were statistically significant (Table 3 ).

In the case of advanced cancer, NL complimented the N stage system for predicting the limit of DFS. For example, the DFS ( $p=0.001)$ of the AL group of CRC, the DFS $(p<0.001)$ of the A group of colon, the DFS ( $\mathrm{p}=0.004$ ) of the AL group of colon, and the DFS ( $\mathrm{p}=0.004)$ of the IAL group of colon showed increased efficacy with the $\mathrm{N}$ stage system (Table 3 ).

Ogino et al. [5] also concluded that smaller LR was associated with improved survival in stage III and stage IV using LR of $<0.2,0.2-0.39$, and $\geq 0.4$. Ceelen et al. [6], who analyzed 16 studies, including 33,984 patients with stage III and IV CRC, identified LR as an independent prognostic factor for patients with stage III cancer of the colon or rectum. The prognostic separation obtained by LR was superior to that of the number of positive nodes ( $\mathrm{N}$ stage). We analyzed the LR using the following three criteria: first, $<0.2,0.2-0.5$, and $\geq 0.5$; second, $\leq 0.17,0.17-0.41,0.41-0.7$, and $>0.7$; third, $<0.2$, $0.2-0.4$, and $>0.4$. We described only the first criteria because it was the most statistically significant in our data.

The APL was defined as the node nearest the point of ligature of the main vascular pedicle [7,14]. Malassagne et al. [7] concluded that the involvement of APLs has a significant effect on colon cancer prognosis. Kang et al. [15] concluded that the presence of inferior mesenteric artery lymph node metastasis should be considered a predictive factor for high systemic recurrence in sigmoid and rectal cancer, and it should be treated and followed up. However, Yi et al. [16] concluded that apical node metastasis is not a poor prognostic factor for stage III sigmoid colon or rectal cancer after high ligation. Interestingly, Huh et al. [17] reported that in patients with pN1 tumors, OS and DFS did not differ significantly according to the distribution of lymph node metastases (lymph node; i.e., APL metastases). However, for patients with pN2 tumors, the OS and DFS curves among the distribution of lymph node metastasis groups were significantly different ( $p<0.001$ and $p<0.001$, respectively) [17]. In granular analysis of our data, APL was not efficient in the IAL group, but it was statistically valuable for predicting OS for colon cancer and DFS for rectal cancer in the stage III and advanced cancer AL group.

\section{Conclusion}

Our study indicates that various lymph node analysis methods complement the existing $\mathrm{N}$ stage. In the future, we plan to determine how to improve the $\mathrm{N}$ stage system based on the $\mathrm{T}$ stage.

\section{Conflicts of Interest}

Conflict of interest relevant to this article was not reported. 


\section{References}

1. Townsend CM Jr, Beauchamp RD, Evers BM, Mattox KL. Sabiston textbook of surgery: the biological basis of modern surgical practice. 19th ed. Philadelphia, PA: Elsevier/Saunders; 2012.

2. Brunicardi FC, Andersen DK, Billiar TR, Dunn DL, Hunter JG, Matthews JB, et al. Schwartz's principles of surgery. 9th ed. New York: McGraw-Hill Education; 2010.

3. Baxter NN, Virnig DJ, Rothenberger DA, Morris AM, Jessurun J, Virnig BA. Lymph node evaluation in colorectal cancer patients: a population-based study. J Natl Cancer Inst. 2005;97: 219-25.

4. Chang GJ, Rodriguez-Bigas MA, Skibber JM, Moyer VA. Lymph node evaluation and survival after curative resection of colon cancer: systematic review. J Natl Cancer Inst. 2007; 99:433-41.

5. Ogino S, Nosho K, Irahara N, Shima K, Baba Y, Kirkner GJ, et al. Negative lymph node count is associated with survival of colorectal cancer patients, independent of tumoral molecular alterations and lymphocytic reaction. Am J Gastroenterol. 2010;105:420-33.

6. Ceelen W, Van Nieuwenhove Y, Pattyn P. Prognostic value of the lymph node ratio in stage III colorectal cancer: a systematic review. Ann Surg Oncol. 2010;17:2847-55.

7. Malassagne B, Valleur P, Serra J, Sarnacki S, Galian A, Hoang C, et al. Relationship of apical lymph node involvement to survival in resected colon carcinoma. Dis Colon Rectum. 1993;36: 645-53.

8. Compton CC, Fielding LP, Burgart LJ, Conley B, Cooper HS, Hamilton SR, et al. Prognostic factors in colorectal cancer. College of American Pathologists Consensus Statement 1999. Arch Pathol Lab Med. 2000;124:979-94.
9. Nedrebo BS, Soreide K, Nesbakken A, Eriksen MT, Soreide JA, Korner $\mathrm{H}$, et al. Risk factors associated with poor lymph node harvest after colon cancer surgery in a national cohort. Colorectal Dis. 2013;15:e301-8.

10. Stocchi L, Fazio VW, Lavery I, Hammel J. Individual surgeon, pathologist, and other factors affecting lymph node harvest in stage II colon carcinoma. is a minimum of 12 examined lymph nodes sufficient? Ann Surg Oncol. 2011;18:405-12.

11. Porter GA, Urquhart R, Bu J, Johnson P, Grunfeld E. The impact of audit and feedback on nodal harvest in colorectal cancer. BMC Cancer. 2011;11:2.

12. Engstrom PF, Arnoletti JP, Benson AB 3rd, Chen YJ, Choti MA, Cooper HS, et al. NCCN Clinical Practice Guidelines in Oncology: colon cancer. J Natl Compr Canc Netw. 2009;7:778831.

13. Johnson PM, Porter GA, Ricciardi R, Baxter NN. Increasing negative lymph node count is independently associated with improved long-term survival in stage IIIB and IIIC colon cancer. J Clin Oncol. 2006;24:3570-5.

14. Grinnell RS. Lymphatic metastases of carcinoma of the colon and rectum. Ann Surg. 1950;131:494-506.

15. Kang J, Hur H, Min BS, Kim NK, Lee KY. Prognostic impact of inferior mesenteric artery lymph node metastasis in colorectal cancer. Ann Surg Oncol. 2011;18:704-10.

16. Yi JW, Lee TG, Lee HS, Heo SC, Jeong SY, Park KJ, et al. Apical-node metastasis in sigmoid colon or rectal cancer: is it a factor that indicates a poor prognosis after high ligation? Int J Colorectal Dis. 2012;27:81-7.

17. Huh JW, Kim YJ, Kim HR. Distribution of lymph node metastases is an independent predictor of survival for sigmoid colon and rectal cancer. Ann Surg. 2012;255:70-8. 